\title{
News on fluctuations and correlations from the NA61/SHINE experiment
}

\author{
Maja Maćkowiak-Pawłowska \\ for the NA61/SHINE Collaboration, ${ }^{1, *}$ \\ ${ }^{1}$ Faculty of Physics, Warsaw University of Technology, Koszykowa 75, 00-662 Warsaw
}

\begin{abstract}
In this contribution the latest NA61/SHINE results on fluctuations and correlations from the $\mathrm{p}+\mathrm{p}, \mathrm{Be}+\mathrm{Be}$, and $\mathrm{Ar}+\mathrm{Sc}$ energy scans will be presented. The NA61 experimental results will be compared with existing NA49 data and with model predictions.
\end{abstract}

\section{Introduction}

Heavy ion collisions are the only controllable way to test non-perturbative regime of strong interactions. In particular, they allow to study the phase diagram of strongly interacting matter and may give an important information to understand its nature.

The NA61/SHINE experiment [1] is a fixed target large acceptance spectrometer located in the North Area of CERN. It studies hadron+hadron, hadron+ion and ion+ion collisions at the SPS energy range (from $13 A \mathrm{GeV} / \mathrm{c}$ up to $400 A \mathrm{GeV} / \mathrm{c}$ for protons and up to $158 A \mathrm{GeV} / \mathrm{c}$ for heavy ions). It's energy range corresponds to region of phase diagram, where the onset of deconfinement is located. Predicted critical point of strongly interacting matter, i.e. place where transition from hadrons to quarks changes from first order phase transition to smooth cross-over, may also be available in this energy range [2].

Figure 1 (left) shows NA61/SHINE detector configuration. The NA61/SHINE consists of four large volume time projection chambers. First two, so-called Vertex TPCs are located in a magnetic field. Downstream of the beam are another two large TPCs, so-called MTPCL and MTPC-R, which improve the quality of the measured tracks. In order to improve particle identification behind TPCs are two Time-Of-Flight walls (ToF-F was not used in the presented analysis). Centrality of a registered collision can be obtained via energy of projectile spectators which is registered in the Projectile Spectator Detector (PSD). The PSD calorimeter consists of 44 modules which cover a transverse area of $120 \times 120 \mathrm{~cm}^{2}$. The central part of the PSD consists of 16 small modules and the outer part consists of 28 large modules. Such fine transverse segmentation decreases the spectator occupancy in one module and improves the reconstruction of the reaction plane. For details see Ref. [3].

\section{Fluctuation measures}

A specific property of the critical point, the increase in the correlation length $\xi$, makes fluctuations its basic signal [4]. Fluctuations at the critical point are expected to increase as

\footnotetext{
*e-mail: majam@if.pw.edu.pl
} 

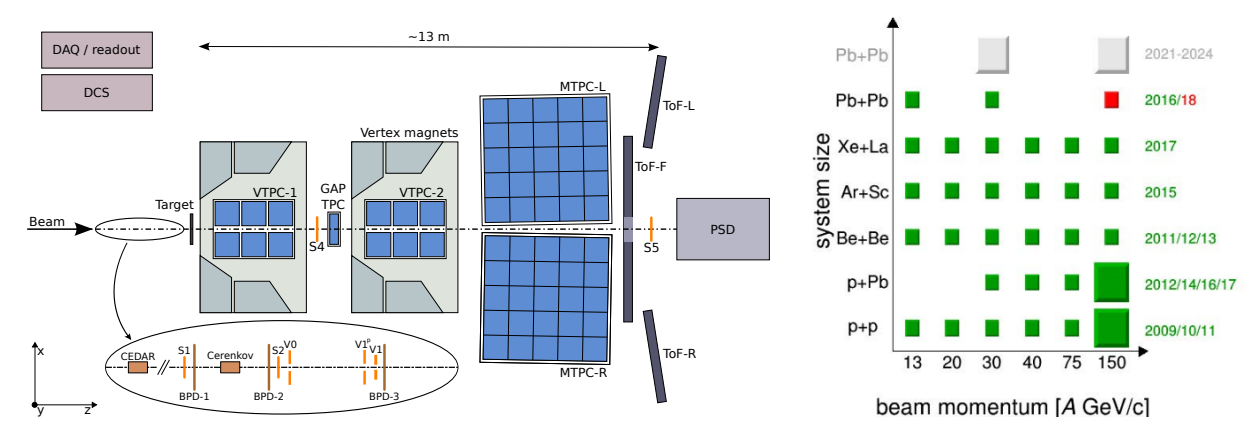

Figure 1. Left: NA61/SHINE detector configuration. For details see text. Right: Data taking status of the strong interaction program of NA61/SHINE

(approximately) $\xi^{2}$ for the variance (second moment) of event-by-event observables such as multiplicities or mean transverse momenta of particles. Thus, the search for CP requires comparison of fluctuation signal measured at different energies and systems such as in the system size - energy scan of NA61/SHINE (Fig. 1 right). In order to compare two different systems one must take into account difference in system size and its fluctuations. Quantities which are insensitive to system size and its fluctuations are called strongly intensive [5]. Two such quantities are used to study $P_{T}-N$ fluctuations [6]:

$$
\begin{array}{r}
\Delta\left[P_{T}, N\right]=\frac{1}{\omega\left[p_{T}\right]\langle N\rangle}\left(\langle N\rangle \omega\left[P_{T}\right]-\left\langle P_{T}\right\rangle \omega[N]\right), \\
\Sigma\left[P_{T}, N\right]=\frac{1}{\omega\left[p_{T}\right]\langle N\rangle}\left(\langle N\rangle \omega\left[P_{T}\right]+\left\langle P_{T}\right\rangle \omega[N]-2 \operatorname{cov}\left(P_{T}, N\right)\right),
\end{array}
$$

where $P_{T}=\sum_{i=1}^{N} p_{T i}$ and $N$ are extensive quantities $(\sim V), \omega\left[p_{T}\right]=\frac{\operatorname{Var}\left[p_{T}\right]}{\left\langle p_{T}\right\rangle}-$ scaled variance of inclusive $p_{T}$ distribution. In addition to being insensitive to system size and its fluctuations $\Sigma\left[P_{T}, N\right]$ and $\Delta\left[P_{T}, N\right]$ provide good scale, namely,

- $\Delta\left[P_{T}, N\right]=\Sigma\left[P_{T}, N\right]=1$ for independent particle production model

- $\Delta\left[P_{T}, N\right]=\Sigma\left[P_{T}, N\right]=1$ for the ideal Boltzmann gas in GCE and $\mathrm{CE}^{1}$

- $\Delta\left[P_{T}, N\right]=\Sigma\left[P_{T}, N\right]=0$ in the absence of fluctuations

The NA61/SHINE analyzed $\Delta\left[P_{T}, N\right]$ and $\Sigma\left[P_{T}, N\right]$ in $\mathrm{p}+\mathrm{p}, \mathrm{Be}+\mathrm{Be}$ and $\mathrm{Ar}+\mathrm{Sc}$ most central collisions [7]. Figure 2 presents obtained dependencies. For all considered energies and systems $\Delta\left[P_{T}, N\right]<1$ and $\Sigma\left[P_{T}, N\right]>1$. Observed behavior is probably caused by BoseEinstein statistics and negative correlation of $P_{T} / N$ vs $N$ [8]. No anomaly attributable to the $\mathrm{CP}$ is visible. Fluctuations described by higher, non-Gausian, moments of considered distributions are under studies. They were already obtained for multiplicity of $h^{-}$and netcharge in $\mathrm{p}+\mathrm{p}$ interactions [9].

$P_{T}-N$ analysis were extended to studies of the pseudorapidity dependence of strongly intensive observables in order to probe different values of baryochemical potential [10]. The preliminary results on pseudorapidity dependence of $\Delta\left[P_{T}, N\right]$ in $\mathrm{Be}+\mathrm{Be}$ and $\mathrm{p}+\mathrm{p}$ interactions at $150 / 158 \mathrm{~A} \mathrm{GeV} / \mathrm{c}$ is shown in Fig. 3. In both reactions $\Delta\left[P_{T}, N\right]$ changes monotonously for the data in contrast to the EPOS1.99 results for $\Delta\left[P_{T}, N\right]$ which show a minimum for intermediate $\eta$ window width and lay significantly above the measurements.

\footnotetext{
${ }^{1} \Delta\left[P_{T}, N\right]$ and $\Sigma\left[P_{T}, N\right]$ are independent of material conservation laws
} 

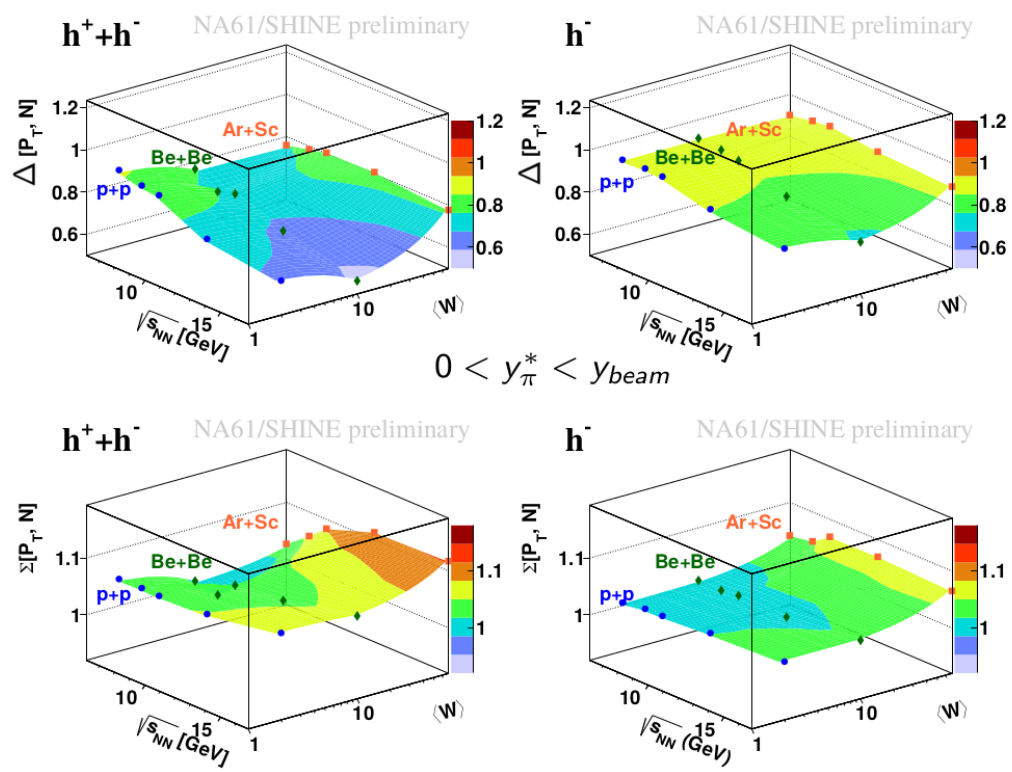

Figure 2. $\Delta\left[P_{T}, N\right]$ and $\Sigma\left[P_{T}, N\right]$ energy and system size dependence in $\mathrm{p}+\mathrm{p}, \mathrm{Be}+\mathrm{Be}$ and $\mathrm{Ar}+\mathrm{Sc}$ central collisions at beam momenta $20-150 / 158 A \mathrm{GeV} / \mathrm{c}$.
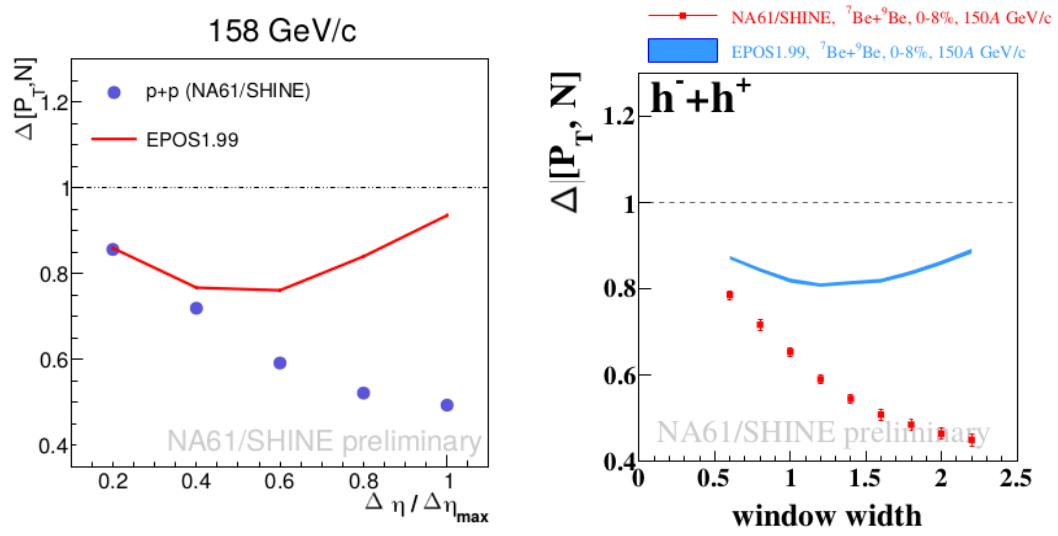

Figure 3. The pseudorapidity dependence of $\Delta\left[P_{T}, N\right]$ in $\mathrm{p}+\mathrm{p}$ interactions at $158 \mathrm{~A} \mathrm{GeV} / \mathrm{c}$ (left) and in $\mathrm{Be}+\mathrm{Be}$ collisions at $150 \mathrm{~A} \mathrm{GeV} / \mathrm{c}$ (right). For details on p+p see Ref. [11] and on Be+Be Ref. [12]

\section{Intermittency}

In search of $\mathrm{CP}$ a powerful tool is proton intermittency which should follow power-law fluctuations near CP. It can be checked by scaling of $2^{\text {nd }}$ factorial moments with cell size. The $2^{\text {nd }}$ factorial moment is defined as:

$$
F_{2}(M)=\left\langle\frac{1}{M^{2}} \sum_{i=1}^{M^{2}} n_{i}\left(n_{i}-1\right)\right\rangle\left\langle\left\langle\frac{1}{M^{2}} \sum_{i=1}^{M^{2}} n_{m}\right\rangle^{2},\right.
$$




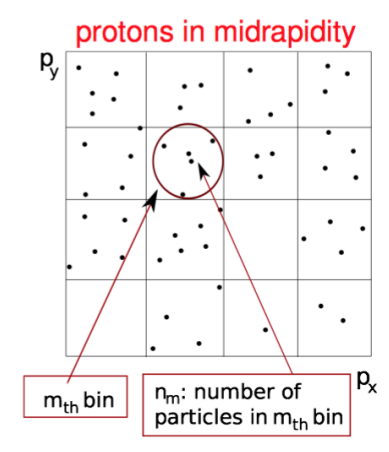

Figure 4. Definition of cells in the proton intermittency analysis in Be+Be at $150 \mathrm{~A} \mathrm{GeV/c}$

where $\langle\ldots\rangle$ indicates averaging over events, $m$ is number of a given cell from $M$ cells dividing momentum space. Illustration of the division into $M$ cells is presented in Fig. 4.

For critical system the predicted power of $F_{2} \sim\left(M^{2}\right)^{\phi_{2, c r}}$ is $\phi_{2, c r}=5 / 6$ [13]. In a noisy system such as real heavy ion collision one needs to subtract background moments with mixed events. Then, correlator $\Delta F_{2}(M)=F_{2}^{\text {data }}-F_{2}^{\text {mixed }}$ should scale according to power-law for $M \gg 1$.

The NA61/SHINE experiment analyzed proton intermittency in $\mathrm{Be}+\mathrm{Be}$ interactions at $150 \mathrm{~A} \mathrm{GeV/c}$ beam momentum[14]. The subtracted correlator dependence on $M^{2}$ is show in Fig. 5 (top). The obtained results are compared to the NA49 results calculated in $\mathrm{Si}+\mathrm{Si}$ at $158 \mathrm{~A} \mathrm{GeV} / \mathrm{c}$ interactions [15], which show power-law fluctuations (Fig. 5 bottom). No intermittency behavior in $\mathrm{Be}+\mathrm{Be}$ at $150 \mathrm{~A} \mathrm{GeV} / \mathrm{c}$ is observed. Comparison with the Critical Monte Carlo (CMC) [13] sets critical component upper limit at $\sim 0.3 \%$. Analysis of other systems and energies are coming soon.

\section{Bose-Einstein correlations}

The CP can be also searched for by the critical exponent $\eta$ which is related to spacial correlations. At the CP the spatial correlation function becomes a power-law $\sim r^{-(d-2+\eta)}$, where $d$ represents the number of dimensions. The predicted value of $\eta$ at the CP is 0.03631 [16]. The spatial momentum correlations can be studied by measuring Bose-Einstein correlations with the use of the HBT method. In case of heavy ions the analyzed correlations are momentum correlations with correlation functions defined as:

$$
C(q) \cong 1+|\tilde{S}|^{2}
$$

where $\tilde{S}$ is the Fourier transform of normalized size distribution, $S(r)$. Usually, the assumed shape of the source is Gaussian but alternate approach may be more realistic. For example, the expanding medium increases the mean free path which may lead to anomalous diffusion and Lévy distributed sources. The Lévy distribution is defined as:

$$
\mathcal{L}(\alpha, R, r)=\frac{1}{2 \pi} \int d^{3} q e^{i q r} e^{\frac{1}{2}|q R|^{2}},
$$

where $R$ is source size and $\alpha=1$ or 2 reduces Lévy distribution to Cauchy or Gauss distribution. In Ref. [17] $\alpha$ is shown to be identical to the spatial correlation exponent. In general anomalous diffusion leads to $\alpha<2$. 

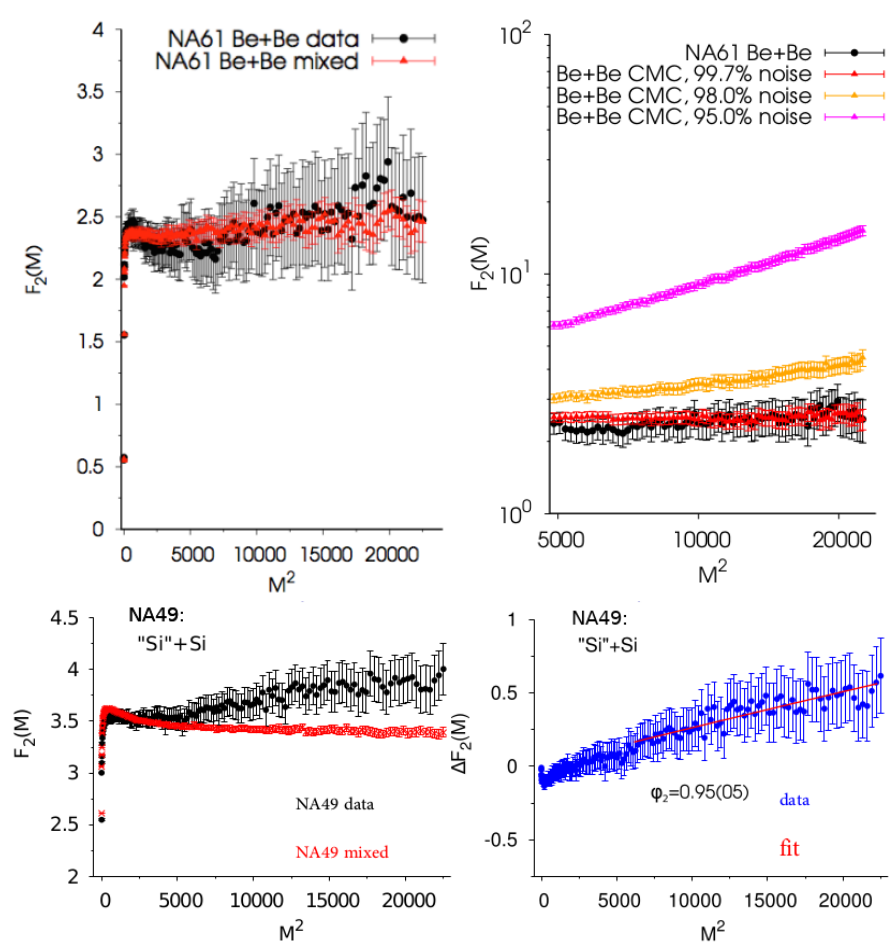

Figure 5. Top: Protons' factorial moment dependence on the number of considered cells $M$ in $\mathrm{Be}+\mathrm{Be}$ at $150 \mathrm{~A} \mathrm{GeV} / \mathrm{c}$ (left) and predictions of CMC model for different fractions of critical component in the system (right) [14]. Bottom: Protons' factorial moment dependence on the number of considered cells $M$ in $\mathrm{Si}+\mathrm{Si}$ at $158 \mathrm{~A} \mathrm{GeV/c} \mathrm{(left)} \mathrm{and} \mathrm{predictions} \mathrm{of} \mathrm{CMC} \mathrm{model} \mathrm{for} 5 \%$ critical component [15]. For details see text.

The presented performance results were obtained in $\mathrm{Be}+\mathrm{Be}$ collisions at $150 \mathrm{~A}$ $\mathrm{GeV} / \mathrm{c}$ [18]. Performance results are shown for centrality higher than $20 \%$ based on the Projectile Spectator Detector. Due to possible trigger bias in this region presented results should be regarded as performance studies. Only negatively charged particles were selected. Even though particles were not identified, the applied cuts results in a relatively clean sample of negatively charged pions since particle contamination from kaons is relatively low (in $\mathrm{p}+\mathrm{p}$ interactions at the same energy the contamination is below $2 \%$ of $\pi$ multiplicity). The repulsion of the same charged particles was taken into account via Coulomb correction (for details see Ref. [18]). The fitted function has three important parameters: $R, \lambda$ and $\alpha$. Figure 6 shows performance plots on the $m_{T}$ dependence of the mentioned parameters in mid-central $\mathrm{Be}+\mathrm{Be}$ collisions at $150 \mathrm{~A} \mathrm{GeV/c}$ beam momentum.

First, the Lévy scale $R$ determines the length of homogeneity. In a simple hydrodynamical picture one obtains $\frac{1}{R^{2}} \propto m_{T}$, which leads to decreasing trend with $m_{T}$ which can be associated with transverse flow. Second, correlation strength $\lambda$ can be interpreted in core-corona picture as:

$$
\lambda=\left(\frac{N_{\text {core }}}{N_{\text {core }}+N_{\text {halo }}}\right)^{2}
$$

where corona consists of primordial particles and products of short lived resonance; and halo contains decays of long lived resonances. This parameter was found to decrease at low $m_{T}$ in 

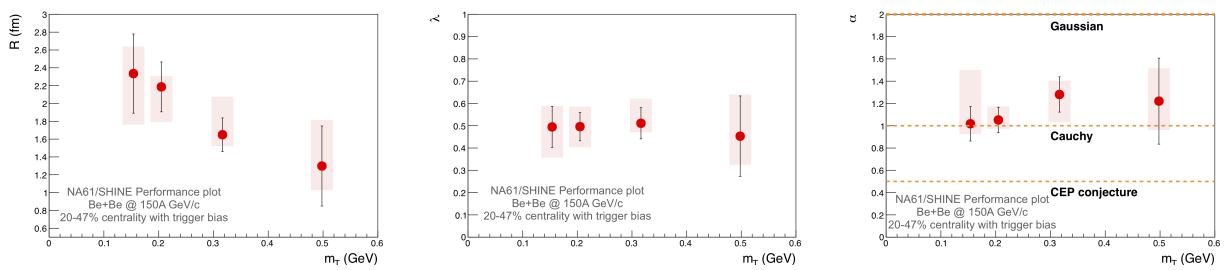

Figure 6. Performance analysis of Bose-Einstein correlations in semi-central $\mathrm{Be}+\mathrm{Be}$ interactions at $150 A \mathrm{GeV} / \mathrm{c}$. The HBT radius (left), $\lambda$ (middle) and $\alpha$ parameters dependence on transverse mass.

$\mathrm{Au}+\mathrm{Au}$ collisions at RHIC which was not observed in $\mathrm{Pb}+\mathrm{Pb}$ collisions at the SPS. Finally, already mentioned the Lévy exponent $\alpha$ may show signal of anomalous diffusion similarly to Ref. [19], where $\alpha \approx 1.2$. In the vicinity of the CP it should decrease further up to 0.5 [20].

Performance plots demonstrates the capabilities of NA61/SHINE to measure BoseEinstein correlations even in a low multiplicity system. It is an important part of search for the critical point and studying other system's properties such as radial flow, etc.

\section{Flow}

The NA61/SHINE as a fixed target experiment allows to extend flow measurements available from the STAR RHIC beam energy scan (BES) program [21] to a wide rapidity range up to the forward region where projectile nucleon spectators appear. Due to capabilities of the Projectile Spectator Detector it is possible to estimate the reaction plane by deflection of projectile spectators in the course of the collision. First such measurements were performed in $\mathrm{Pb}+\mathrm{Pb}$ collisions at $30 \mathrm{~A} \mathrm{GeV} / \mathrm{c}$ beam momentum collected in 2016 [22]. The available statistics is $1.1 \mathrm{M}$ events for the minimum bias trigger and $0.6 \mathrm{M}$ events of $0-15 \%$ centrality.

The Projectile Spectator Detector is sensitive to spectator fragments (the outer modules are also sensitive to produced particles) and it is used for centrality selection of events and estimation of the reaction plane. Figure 7 (left) shows PSD energy for central and minimum bias events. In order to determine reaction plane PSD was divided into 3 groups called PSD1,PSD2 and PSD3 with acceptance coverage $\eta \in\{(5.1, \infty),(4.4,5.1),(4.0,4.4)\}$. Azimuthal asymmetry of the measured distributions is described with two dimensional flow vectors $\overrightarrow{q_{n}}$ and $\overrightarrow{Q_{1}}$ determined event-by-event from the TPC tracks and PSD subevents:

$$
Q_{1}^{s}=\frac{1}{E_{s}} \sum_{i}^{N_{s}} E_{i} \overrightarrow{n_{i}} ; \quad \overrightarrow{q_{n}}=\frac{1}{M} \sum_{i} M \overrightarrow{u_{n, i}} ; \overrightarrow{u_{n, i}}=\operatorname{conn} \phi, \operatorname{sinn} \phi,
$$

where the unit vector $\overrightarrow{n_{i}}$ points to the center of $i$-th PSD module, $E_{i}$ is the energy deposited in the $i$-th module, $N_{s}$ is the number of modules and $E_{s}=\sum_{i}^{N_{s}} E_{i}$ is energy of a whole part of PSD (i.e. PSD1,PSD2 or PSD3). For each particle track $i$ reconstructed with the TPC a $n$-th harmonic unit vector $\overrightarrow{u_{n, i}}$ is defined. The $\overrightarrow{q_{n}}$-vectors were calculated for charged pions and protons in transverse momentum bins (Eq. 7), where $M$ is number of particles in a given bin.

The flow harmonics $v_{n}$ can be obtained using the scalar product method:

$$
v_{1}^{\alpha}\{A ; B, B\}=\frac{2\left\langle q_{1, \alpha} Q_{1, \alpha}^{A}\right\rangle}{R_{1, \alpha}^{A}\{B, C\}} ; \quad v_{2}^{\alpha \beta \gamma}\{A, B ; C\}=\kappa_{\alpha \beta \gamma} \frac{4\left\langle q_{2, \alpha} Q_{1, \beta}^{A} Q_{1, \gamma}^{B}\right\rangle}{R_{1, \alpha}\{B, C\} R_{1, \gamma}^{B}\{A, C\}},
$$



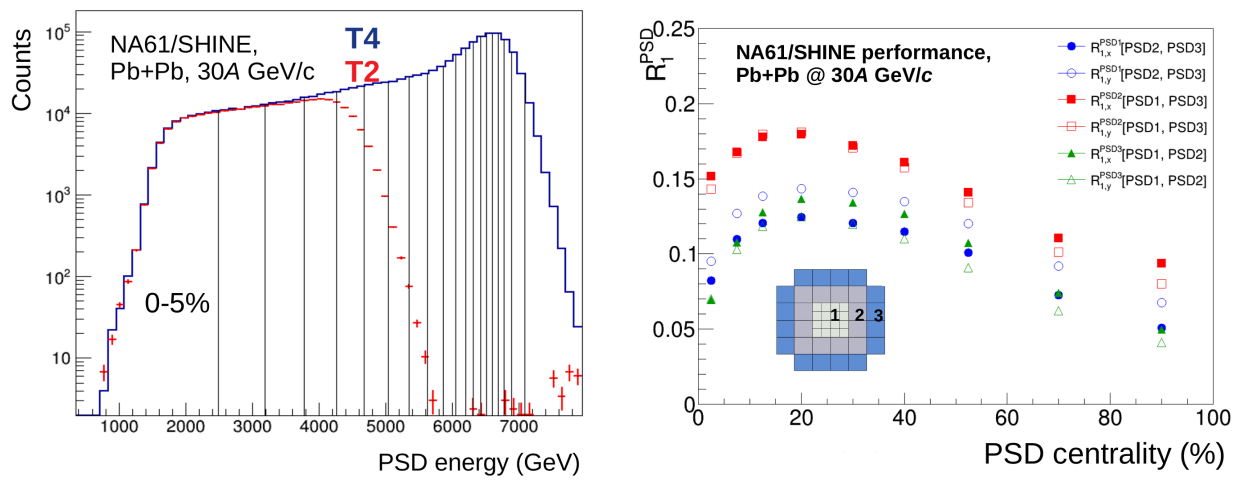

Figure 7. Left: Central and minimum bias trigger in $\mathrm{Pb}+\mathrm{Pb}$ collisions at $30 \mathrm{~A} \mathrm{GeV} / \mathrm{c}$. Right: Resolution of the correction factors $R_{1}^{A}\{B, C\}$ obtained for PSD subgroups using 3-subevent method. For details see text.

where for $v_{1} \alpha=x, y$, for $v_{2} k a p p a_{\alpha \beta \gamma}=1$ for $(\alpha, \beta, \gamma)=\{(x, x, x),(y, x, y),(y, y, x)\}$ and $\kappa_{\alpha \beta \gamma}=-1(\alpha, \beta, \gamma)=\{(x, y, y)\} . \vec{Q}$-vector correction factors $R_{1, \alpha}^{A}\{B, C\}$ are calculated using the 3-subevent method:

$$
R_{1, \alpha}^{A}\{B, C\}=\sqrt{2 \frac{\left\langle Q_{1, \alpha}^{A} Q_{1, \alpha}^{B}\right\rangle\left\langle Q_{1, \alpha}^{A} Q_{1, \alpha}^{C}\right\rangle}{\left\langle Q_{1, \alpha}^{B} Q_{1, \alpha}^{C}\right\rangle}}
$$

Imperfect acceptance of the aziumthal angle and detector efficiency was corrected using a Mont-Carlo simulation of the detector response and DCM-QGSM model [23]. Figure 7 (right) shows the resolution of the correction factors $R_{1}$ for $x$ an $y$ using all three sub PSD parts. Obtained results are corrected for detector non-uniformity. No corrections for secondary interactions and weak decays were applied. Comparison of $v_{1}$ and $v_{2}$ coming from different subgroups of PSD are consistent within statistical uncertainties. For $v_{1}$ only $x$ component is used and for $v_{2}$ only average of $(y, x, y)$ and $(y, y, x)$ are used due to the smallest statistical uncertainty. Figure 8 (left) shows directed flow as a function of $p_{T}$ and comparison with a new analysis of $\mathrm{NA} 49$ data for $\mathrm{Pb}+\mathrm{Pb}$ collisions at $30 \mathrm{~A} \mathrm{GeV} / \mathrm{c}$ using forward calorimeters (VCal and RCal) for projectile spectator plane estimation [24]. Results of NA61/SHINE and NA49 are in agreement within statistical uncertainty. Figure 8 (right) presents $v_{1}$ for $\pi^{-}$, $\pi^{+}$and $p$ as a function of centrality in PSD. Slope of $v_{1}$ pions is always negative or close to 0 . Slope of protons changes sign close to $50 \%$ centrality.

Comparison of $v_{2}$ for different particle species as a function of $p_{T}$ (Fig. 9 left) shows mass dependence indicating radial flow. Comparison of protons $v_{2}$ with results of the STAR experiment are shown in Fig. 9 (right). The obtained signal is similar for central and peripheral collisions but for semi-central collisions there is a clear discrepancy between NA61/SHINE and STAR results.

In future, measurements will be extended to other collision energies ( $13 \mathrm{~A}$ and $150 \mathrm{~A}$ $\mathrm{GeV} / \mathrm{c}$ ) and collisions of other systems such as $\mathrm{Xe}+\mathrm{La}, \mathrm{Ar}+\mathrm{Sc}$, and $\mathrm{Be}+\mathrm{Be}$ collisions, which are available from the system size scan of the NA61/SHINE program. 

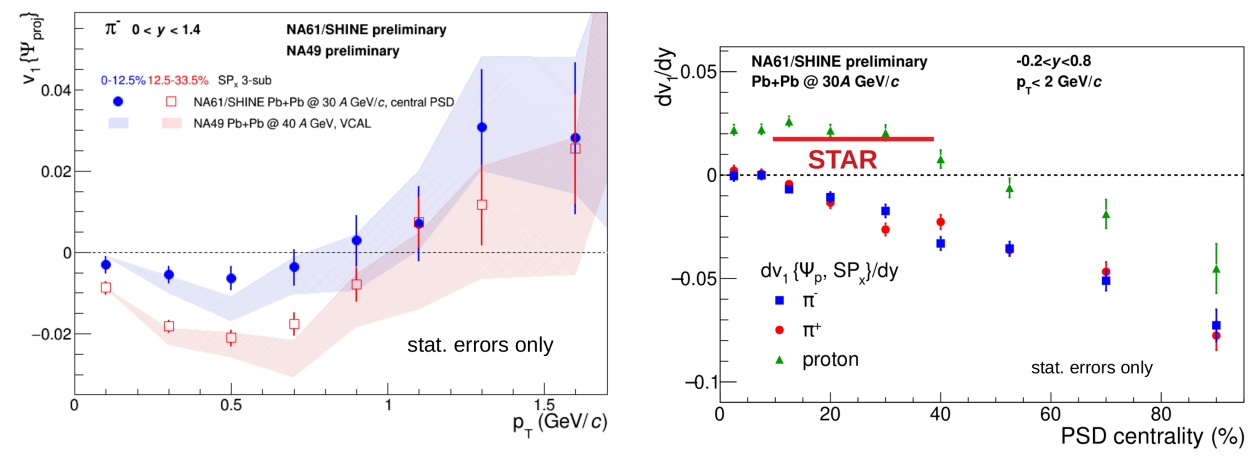

Figure 8. Left: Dependence of $v_{1}$ on transverse momentum measured by NA61/SHINE and NA49 [24] in $\mathrm{Pb}+\mathrm{Pb}$ collisions at $30 A \mathrm{GeV} / \mathrm{c}$. Right: Resolution of the correction factors $R_{1}^{A}\{B, C\}$ obtained for PSD subgroups using 3-subevent method. For detials see text.
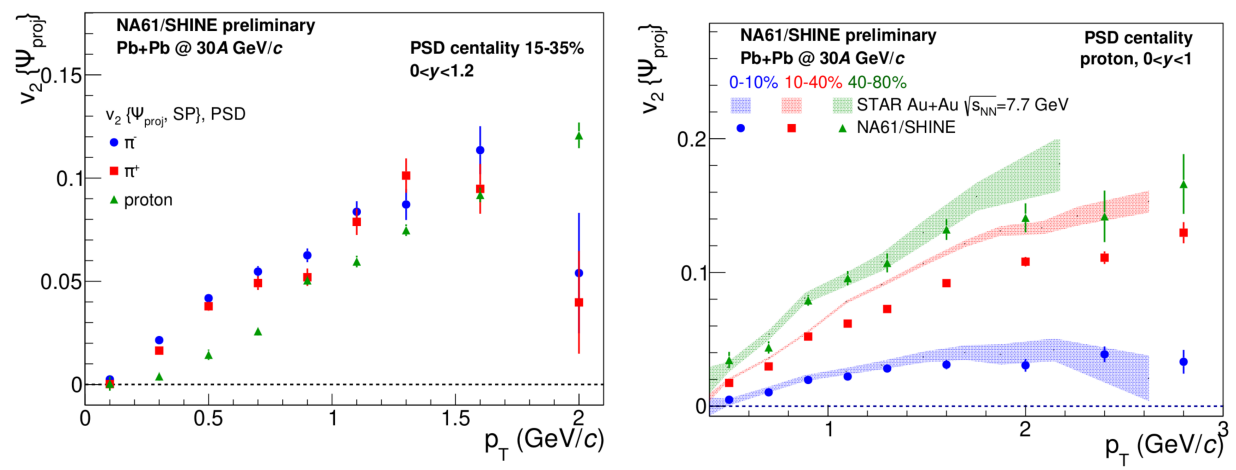

Figure 9. Left: Transverse momentum dependence of $v_{2}$ for different particle types in $\mathrm{Pb}+\mathrm{Pb}$ collisions at $30 \mathrm{~A} \mathrm{GeV} / \mathrm{c}$. Right: Transverse momentum dependence of protons's $v_{2}$ in $\mathrm{Pb}+\mathrm{Pb}$ interactions measured by NA61/SHINE and in Au+Au interactions measured by STAR.

\section{Summary}

Currently, NA61/SHINE is the only CERN experiment providing hadron production data as a function of energy, reaction type and centrality at the SPS energy range. This is an unique opportunity to study correlations and fluctuations in a systematic way which allows for an extensive search of the CP. In particular, intermitency analysis of $\mathrm{Ar}+\mathrm{Sc}$ collisions and analysis of higher, non-Gausian fluctuations of multiplicity and net-charge are particulary interesting. New results on flow in $\mathrm{Pb}+\mathrm{Pb}$ collision add a new set of tools to study heavy ion collisions.

This work was partially supported by the Polish National Center for Science grants 2016/21/D/ST2/01983 and 2015/18/M/ST2/00125. 


\section{References}

[1] N. Antoniou et al. [NA49-future Collaboration], CERN-SPSC-2006-034, CERN-SPSCP-330, CERN-SPSC-2012-022, SPSC-P-330-ADD-6.

[2] K. Grebieszkow for the NA49 Coll., Nucl. Phys. A830, 547C (2009).

[3] N. Abgrall et al. [NA61 Collaboration], JINST 9, P06005 (2014) [arXiv:1401.4699 [physics.ins-det]].

[4] M. A. Stephanov, Acta Phys. Polon. B 35, 2939-2962 (2004)

[5] M. I. Gorenstein and M. Gazdzicki, Phys. Rev. C 84, 014904 (2011) doi:10.1103/PhysRevC.84.014904 [arXiv:1101.4865 [nucl-th]].

[6] M. Gazdzicki, M. I. Gorenstein and M. Mackowiak-Pawlowska, Phys. Rev. C 88, no. 2, 024907 (2013) doi:10.1103/PhysRevC.88.024907 [arXiv:1303.0871 [nucl-th]].

[7] E. Andronov, Acta Phys. Polon. Supp. 10, 449-453 (2017)

[8] M. I. Gorenstein and K. Grebieszkow, Phys. Rev. C 89, no. 3, 034903 (2014) doi:10.1103/PhysRevC.89.034903 [arXiv:1309.7878 [nucl-th]].

[9] M. Maćkowiak-Pawłowska [NA61/SHINE Collaboration], Acta Phys. Polon. Supp. 10, 657 (2017) doi:10.5506/APhysPolBSupp.10.657 [arXiv:1610.03838 [nucl-ex]].

[10] J. Brewer, S. Mukherjee, K. Rajagopal, Y. Yin, arXiv:1804.10215.

[11] D. Prokhorova, Quarks 2018, presentation.

[12] E. Andronov [NA61/SHINE Coll.], [arXiv:1807.10737 [nucl-ex]].

[13] N. G. Antoniou et al., Phys. Rev. Lett. 97, 032002 (2006)

[14] N. Davis [NA61/SHINE Coll.], Quark Matter 2018, poster.

[15] T. Anticic et al. [NA49 Coll.], Eur. Phys. J. C 75, no. 12, 587 (2015) doi:10.1140/epjc/s10052-015-3738-5 [arXiv:1208.5292 [nucl-ex]].

[16] S. El-Showk et al., Journal of Statistical Physics 157, 869 (2014)

[17] T. Csörgö, S. Hegyi and W.A. Zajc, Eur. Phys. J. C36, 67 (2004)

[18] B. Porfy [NA61/SHINE Coll.], arXiv:1811.05262 [nucl-ex]

[19] A. Adare et al. [PHENIX Coll.], Phys. Rev. C97, 064911 (2018)

[20] H. Rieger, Phys. Rev. B52, 6659 (1995)

[21] L. Adamczyk, et al. [STAR Collaboration], Phys. Rev. Lett. 112, 162301 (2014)

[22] V. Klochkov, I. Selyuzhenkov [NA61/SHINE Coll.], arXiv:1810.07579 [nucl-ex]

[23] I. Selyuzhenkov, S. Voloshin, Phys. Rev. C77, 034904 (2008)

[24] O. Golosov, V. Klochkov, I. Selyuzhenkov and E. Kashirin [NA49 Coll.], QM2018 poster \#367. 